\title{
Thermal width of the Higgs boson in hot QCD matter
}

\author{
Jacopo Ghiglieri ${ }^{*}$ and Urs Achim Wiedemann ${ }^{\dagger}$ \\ Theoretical Physics Department, CERN, CH-1211 Genève 23, Switzerland
}

(Received 17 January 2019; published 8 March 2019)

\begin{abstract}
Following Caron-Huot and combining results for the thermal dependence of spectral functions at large timelike momenta, we write an explicit expression for the thermal width of the Higgs boson to $\mathcal{O}\left(\alpha_{\mathrm{s}}\right)$ for $T \ll M_{H}$. It is an $\mathcal{O}\left(\alpha_{\mathrm{s}}\left(\frac{T}{M_{H}}\right)^{4}\right)$ correction for $H \rightarrow g g$ and $H \rightarrow q \bar{q}$. We also compile corresponding results for the thermal width of the $Z$-boson, and we recall which generic structures of the field theory, accessible via the operator product expansion, fix the $\frac{T}{M}$-dependence of the decay of heavy particles.
\end{abstract}

DOI: 10.1103/PhysRevD.99.054002

\section{INTRODUCTION}

In this paper we derive the thermal correction $\delta \Gamma_{H}$ of the width of the Higgs boson to first order in the strong coupling constant $\alpha_{s}$ and for temperatures $T$ of the QCD plasma that are parametrically lower than the Higgs mass, $M_{H} \gg T$. We do so since we could not find an explicit expression for $\delta \Gamma_{H}$ in the literature when discussing the (im)possibility of observing thermal corrections to Higgs branching ratios at future multi- $\mathrm{TeV}$ heavy ion collider experiments [1-3]. As we explain below, $\delta \Gamma_{H}$ can be obtained essentially from combining limiting cases of several spectral functions whose derivation has been described in detail [4-6]. We believe this to be known to a small group of experts in thermal field theory, and the novelty of the present work thus resides mainly in making this expert knowledge explicit.

For thermal corrections to the Higgs width in a QCD plasma, the branching into final states without color charge (such as $H \rightarrow Z Z \rightarrow 4 l$ ) is clearly unimportant. The decay processes relevant for the following are therefore determined by the electroweak interaction of the Higgs to quarks,

$$
\mathcal{L}_{H q}=-S \frac{H}{v}, \quad S \equiv m_{q} \bar{\psi}_{q} \psi_{q},
$$

and by the corresponding coupling of the Higgs to gluons. Here, $v \cong 246 \mathrm{GeV}$ denotes the Higgs vacuum expectation value. The dominant contribution to $H \rightarrow g g$ proceeds via a

\footnotetext{
*jacopo.ghiglieri@cern.ch

urs.wiedemann@cern.ch
}

Published by the American Physical Society under the terms of the Creative Commons Attribution 4.0 International license. Further distribution of this work must maintain attribution to the author(s) and the published article's title, journal citation, and DOI. Funded by SCOAP . top quark loop. We work in the limit $m_{t} \gg M_{H}$ in which this interaction is given by the Higgs effective field theory Lagrangian [7]

$$
\begin{aligned}
\mathcal{L}_{H g}^{\mathrm{eff}} & =-C_{H g} \frac{H}{v} \mathcal{O}_{H g}, \\
\mathcal{O}_{H g} & \equiv-\frac{1}{4} F_{\mu \nu}^{a} F^{a \mu \nu}, \\
C_{H g} & =\frac{\alpha_{\mathrm{s}}}{3 \pi}+\mathcal{O}\left(\alpha_{\mathrm{s}}^{2}\right) .
\end{aligned}
$$

For a particle that does not carry charges of the plasma and that couples to currents $J$, the decay widths can be expressed in terms of the corresponding spectral functions

$$
\rho_{J}(K) \equiv \int d^{4} x e^{i K \cdot X}\langle[J(X), J(0)]\rangle
$$

where $K_{\mu}=\left(k_{0}, \vec{k}\right)$ in the rest frame of the QCD plasma. The metric is mostly minus. In particular, the partial decay widths of the Higgs boson relevant for our study are given by

$$
\begin{aligned}
\Gamma_{H \rightarrow q \bar{q}} & =\frac{1}{v^{2}} \frac{1}{2 k^{0}} \rho_{S}(K), \\
\Gamma_{H \rightarrow g g} & =\frac{\alpha_{\mathrm{s}}^{2}}{(3 \pi)^{2} v^{2}} \frac{1}{2 k^{0}} \rho_{\mathcal{O}_{H g}}(K),
\end{aligned}
$$

where $\frac{1}{2 k_{0}}$ is the usual kinematical flux factor and the factors $\frac{1}{v^{2}}, \frac{\alpha_{s}^{2}}{(3 \pi)^{2} v^{2}}$ denote the squares of the couplings of the Higgs boson to the corresponding currents. The widths (6), (7) are thus first order in the electroweak couplings, but the spectral functions $\rho_{J}$ are all orders in $\alpha_{s}$.

The calculation of thermal corrections to $\Gamma_{H \rightarrow q \bar{q}}, \Gamma_{H \rightarrow g g}$ then amounts to determining thermal corrections $\delta \rho_{S}(K)$ 
and $\delta \rho_{\mathcal{O}_{H g}}(K)$ to the vacuum spectral functions $\rho_{S}^{\mathrm{vac}}\left(K^{2}\right)$ and $\rho_{\mathcal{O}_{H g}}^{\mathrm{vac}}\left(K^{2}\right)$. Lorentz invariance of the vacuum implies that the latter can depend only on the scalar $K^{2}=K_{\mu} K^{\mu}$. In contrast, any finite temperature system singles out a rest frame, and the thermal corrections $\delta \rho_{S}$ and $\delta \rho_{\mathcal{O}_{H g}}$ can therefore depend separately on $k_{0}$ and $k \equiv|\vec{k}|$. In the present paper, we focus on the case of a Higgs boson at rest in the plasma, except for a short discussion of boosted Higgs bosons in Sec. II C.

In a QCD plasma, the vacuum branchings $H \rightarrow g g$ and $H \rightarrow q \bar{q}$ are modified already to zeroth order in $\alpha_{s}$, since emission of each final state gluon or final state quark of momentum $k_{g / q}$ is enhanced by a thermal Bose-Einstein $\left(1+f_{B}\left(k_{g}\right)\right)$ or suppressed by a Fermi-Dirac $\left(1-f_{F}\left(k_{q}\right)\right)$ distribution factor, respectively. However, the partons emerging from this two-body decay carry momenta $k_{q / g}=$ $\frac{M_{H}}{2}$ much above the thermal scale. As a consequence, the effects of stimulated emission for the decay into gluons and of Pauli-blocking for the decay into quarks are negligible. To zeroth order in $\alpha_{s}$, thermal corrections to $\Gamma_{H \rightarrow g g}$ and $\Gamma_{H \rightarrow q \bar{q}}$ are kinematically suppressed by multiplicative factors $\exp \left[-\frac{k_{g / q}}{T}\right]=\exp \left[-\frac{M_{H}}{2 T}\right]$.

Processes to first order in $\alpha_{s}$ open up a region of phase space in which thermal corrections are not suppressed in this way. First, to $O\left(\alpha_{s}\right)$, there are real emission contributions, such as $H \rightarrow g g g$ or $H \rightarrow q \bar{q} g$. In these three-body decays, one of the three final state partons can carry a momentum $k_{g / q} \lesssim T$, for which effects of stimulated gluon emission $\propto\left(1+f_{B}\left(k_{g}\right)\right)$ and Pauli-blocked quark emission $\propto\left(1-f_{F}\left(k_{q}\right)\right)$ are not suppressed. Second, there are real absorption contributions in which quarks and gluons from the QCD plasma interact with the vacuum branching process, such as $g H \rightarrow g g, g H \rightarrow q \bar{q}, q H \rightarrow q g$ or $\bar{q} H \rightarrow g \bar{q}$. Third, there are thermal virtual contribution that arise from branching processes which interact on the amplitude level to $O\left(\alpha_{s}\right)$ with partons in the medium, and which interfere with the vacuum contribution in the complex conjugate amplitude. In general, calculations of thermal widths to $O\left(\alpha_{s}\right)$ amount to determining these three classes of contributions consistently in finite temperature field theory. There are two conceptually different approaches for achieving this:

(1) Explicit perturbative calculation of $\delta \Gamma$ in finite temperature $Q C D$. This standard approach is well documented e.g., for calculations of the thermal production of vector bosons [8-13]. It is typically formulated in terms of the two-loop self-energy correction of the propagator of the particle excitation in whose width one is interested. By the optical theorem, the imaginary part of this self-energy corresponds to a sum over different cut contributions that can be identified with the three above-mentioned classes of thermal corrections, namely real emission, real absorption and virtual correction. In practice, all three classes of thermal corrections yield infrared singular expressions, while thermal corrections to the sum of the three contributions are infrared and collinear safe observables [14]. This makes explicit perturbative calculations of $\delta \Gamma$ relatively complex and lengthy.

(2) Calculating $\delta \Gamma$ via the operator product expansion $(O P E)$ of the relevant spectral functions. In general, the OPE relies on a systematic separation of infrared and ultraviolet contributions. As first pointed out by Caron-Huot [4], this approach allows one to determine thermal corrections to spectral functions in the high-energy timelike region $k_{0} \gg T$. For the thermal width of particles whose mass is parametrically larger than the plasma temperature, this allows for a much simplified calculation.

A particularly simple and instructive example is the case of a heavy fermion decaying to a lighter fermion and a scalar in a QED plasma. This process was studied in an explicit perturbative calculation in Ref. [15], presenting explicit IR-regulated results for the real emission, real absorption and virtual correction contributions, and demonstrating the IR-finiteness of the physical width in detail. The same process was studied in Ref. [16] with OPE techniques. (As the decaying particle in this toy model is charged under the gauge group, the techniques of Ref. [4] do not apply directly to this case.)

OPE techniques have been applied also to study the thermal corrections to the decay width of hypothetical heavy right-handed neutrinos in [17] (see also [18] for the same calculation with the explicit method). Furthermore, in the case of heavy right-handed neutrinos an effective field theory approach has been introduced in [19], where the $M \gg T$ expansion is introduced at the Lagrangian level, making the separation of IR and UV extremely transparent (see [20] for a review of these calculations in their physical context).

In the present work, we utilize the OPE approach of Ref. [4] to arrive at an expression for the thermal width of the Higgs boson. In Appendix A, we comment shortly on how these results are connected to results obtained from an explicit perturbative calculation. In Appendix B, we also summarize results for the thermal width of the $Z$-boson.

\section{HIGGS BRANCHING RATIOS FROM KNOWN SPECTRAL FUNCTIONS OF $T^{\mu \nu}$}

For the reader who wants to get to the final result for the thermal width $\delta \Gamma_{H}$ without spending too much time on technical details, we compile in this section what is known about the spectral functions $\rho_{\mathcal{O}_{H g}}(K)$ and $\rho_{S}(K)$ in the asymptotic limit of large $K^{2}$, and we insert this information for $K^{2}=M_{H}^{2}$ into Eqs. (6) and (7) to obtain $\delta \Gamma_{H}$. A more thorough discussion of the theoretical basis of this schematic derivation is deferred to the subsequent section. 


\section{A. $H \rightarrow g g$}

To determine $\rho_{\mathcal{O}_{H g}}(K)$, we exploit that the operator $\mathcal{O}_{H g}$ appears in the trace of the QCD energy-momentum tensor [4]

$$
T_{\mu}^{\mu}=\frac{-b_{0} \alpha_{s}}{2 \pi} \mathcal{O}_{H g}+[\text { fermionic terms }],
$$

where $b_{0}=\left(\frac{11}{3} C_{A}-\frac{4}{3} n_{f} T_{F}\right)$ is the leading coefficient of the $\beta$-function, with $C_{A}=N_{c}=3$ the quadratic Casimir of the adjoint representation and $T_{F}=\frac{1}{2}$. The spectral function of the trace anomaly (8) is the spectral function $\rho_{\zeta}$ of the bulk viscous channel of $T^{\mu \nu}$. In general, due to the fermionic mass contributions, $\rho_{\zeta}$ differs from $\rho_{\mathcal{O}_{H g}}$ not only by a trivial prefactor $\frac{b_{0}^{2} \alpha_{s}^{2}}{(2 \pi)^{2}}$, but also by additional fermionic terms. However, in the OPE of $\rho_{\zeta}$, these fermionic terms are suppressed by additional powers of $m_{q}^{2} / K^{2}$ which render them negligible for our problem. From the result for $\rho_{\zeta}^{\mathrm{vac}}\left(K^{2}\right)$ in Ref. [4], one thus finds

$$
\begin{aligned}
\rho_{\mathcal{O}_{H g}}^{\mathrm{vac}}\left(K^{2}\right) & =\frac{(2 \pi)^{2}}{b_{0}^{2} \alpha_{s}^{2}}\left(\rho_{\zeta}^{\mathrm{vac}}\left(K^{2}\right)+\mathcal{O}\left(\frac{m_{q}^{2}}{K^{2}}\right)\right) \\
& =\frac{d_{A}\left(K^{2}\right)^{2}}{32 \pi}+\mathcal{O}\left(\alpha_{\mathrm{s}}\right)+\mathcal{O}\left(\frac{m_{q}^{2}}{K^{2}}\right),
\end{aligned}
$$

where $d_{A}=N_{c}^{2}-1$ is the dimension of the adjoint representation.

The leading (dimension-four) thermal correction to $\rho_{\zeta}$ was derived in the same Ref. [4] up to an unknown coefficient in front of the trace anomaly that has been determined in Refs. $[5,6]$. Accounting again for the fact that $\delta \rho_{\zeta}(K)$ and $\delta \rho_{\mathcal{O}_{H g}}(K)$ differ by the prefactor $\frac{b_{0}^{2} \alpha_{s}^{2}}{(2 \pi)^{2}}$, these results translate into

$$
\begin{aligned}
\delta \rho_{\mathcal{O}_{H g}}(K)= & \frac{2 \alpha_{\mathrm{s}}}{3} \frac{K_{\mu} K_{\nu}}{K^{2}}\left[2 C_{F} T_{f}^{\mu \nu}-\left(n_{f} T_{F}+\frac{3}{2} b_{0}\right) T_{g}^{\mu \nu}\right] \\
& -\pi T^{\mu}{ }_{\mu},
\end{aligned}
$$

where $T_{g}^{\mu \nu}$ and $T_{f}^{\mu \nu}$ denote the traceless parts of the gluonic and fermionic contributions to $T^{\mu \nu}$, respectively, and $C_{F}=\left(N_{c}^{2}-1\right) /\left(2 N_{c}\right)$ is the quadratic Casimir of the fundamental representation. The temperature dependence of $\delta \rho_{\mathcal{O}_{H g}}(K)$ thus enters via the temperature dependence of the energy-momentum tensor.

\section{Vacuum width from spectral function}

Before turning to a discussion of the thermal corrections, we check the consistency of our starting point by determining the corresponding partial width in vacuum. Plugging Eq. (9) into Eq. (7), we find

$$
\Gamma_{H \rightarrow g g}^{\mathrm{vac}}=\frac{\alpha_{\mathrm{s}}^{2} M_{H}^{3}}{72 \pi^{3} v^{2}}+\mathcal{O}\left(\alpha_{\mathrm{s}}^{3}\right),
$$

which agrees with the expression in the literature [7].

This argument can be extended to next-to-leading-order (NLO). Higher order corrections to the Wilson coefficient $C_{H g}$ can be found in [7,21-24]

$$
C_{H g}=\frac{\alpha_{\mathrm{s}}}{3 \pi}\left\{1+\left(\frac{5}{4} C_{A}-\frac{3}{4} C_{F}\right) \frac{\alpha_{\mathrm{s}}}{\pi}\right\} .
$$

The vacuum pure glue part of the bulk channel spectral function $\rho_{\zeta}$ at NLO can be found e.g., in Ref. [25]. Multiplying this with the prefactor $\frac{b_{0}^{2} \alpha_{s}^{2}}{(2 \pi)^{2}}$ of Eq. (8) yields

$$
\rho_{\mathcal{O}_{H g}}^{\mathrm{vac}, \mathrm{NLO}}\left(K^{2}\right)=\rho_{\mathcal{O}_{H g}}^{\mathrm{vac}}\left(K^{2}\right)\left(1+\frac{\alpha_{\mathrm{s}} N_{c}}{4 \pi} \frac{73}{3}\right) .
$$

Combining these expressions, one finds for the NLO correction to $\Gamma_{H \rightarrow g g}^{\mathrm{vac}}$

$$
\begin{aligned}
& \Gamma_{H \rightarrow g g(g), H \rightarrow g q \bar{q}}^{\mathrm{vac}, \mathrm{NLO}} \\
& \quad=\Gamma_{H \rightarrow g g}^{\mathrm{vac}}\left\{1+\frac{\alpha_{\mathrm{s}}}{4 \pi}\left(2\left(5 N_{c}-3 C_{F}\right)+N_{c} \frac{73}{3}\right)\right\} .
\end{aligned}
$$

This is consistent with the NLO correction factor for the pure glue part, $\left(1+\frac{95}{4} \frac{\alpha_{\mathrm{s}}}{\pi}\right)[7,26]$. Equation (13) contains to $\mathcal{O}\left(\alpha_{\mathrm{s}}\right)$ also a logarithmic term that can be traced back to the $\mathrm{RG}$ evolution of the LO result and that is consistent with $[7,26]$. Indeed, we are following here essentially the logic of Ref. [7]. Our reason for repeating this result is that we take in the following thermal corrections to (13) from published results in which also the vacuum contribution to the spectral function is given. The rederivation of (11) and (14) thus serves as a check that these thermal corrections are used with proper normalization.

\section{Thermal corrections to $\boldsymbol{H} \rightarrow \mathrm{gg}$}

Paralleling the discussion in Sec. II A 1, we obtain the thermal correction to $\Gamma_{H \rightarrow g g}^{\mathrm{vac}}$ from the thermal contribution to the quark and gluon condensates that appear in the OPE of the bulk channel spectral function Eq. (10),

$$
\begin{aligned}
\delta \Gamma_{H \rightarrow g g}= & \frac{\alpha_{\mathrm{s}}^{3}}{81 \pi^{2} v^{2} k^{0}} \frac{3 k_{0}^{2}+k^{2}}{M_{H}^{2}}\left[2 C_{F}\left(\sum_{q \in u d s c}\left\langle T_{f q}^{00}\right\rangle\right)\right. \\
& \left.-\left(\frac{11}{2} C_{A}-n_{f} T_{F}\right)\left\langle T_{g}^{00}\right\rangle\right]+\mathcal{O}\left(\alpha_{\mathrm{s}}^{4}\right) .
\end{aligned}
$$

Here, we have used that in an isotropic medium, the traceless operators $T_{f, g}^{\mu \nu}$ satisfy $T_{f, g}^{i j}=\frac{1}{3} \delta^{i j} T_{f, g}^{00}$ and $T_{f, g}^{o j}=0$. The 
resulting prefactor $\left(3 k_{0}^{2}+k^{2}\right)$ breaks Lorentz invariance since the QCD plasma specifies a thermal rest frame.

In close analogy to the NLO vacuum correction (14) to $\Gamma_{H \rightarrow g g}^{\mathrm{vac}}$, also the $\mathcal{O}\left(\alpha_{\mathrm{s}}\right)$ thermal correction $\delta \Gamma_{H \rightarrow g g}$ contains contributions with a $g q \bar{q}$-vertex. On the one hand, these are the processes $g H \rightarrow q \bar{q}$ and $H \rightarrow g q \bar{q}$ with a thermal gluon and a hard quark-antiquark pair, which give rise to the term $\propto n_{f} T_{F}\left\langle T_{g}^{00}\right\rangle$ in (15). On the other hand, there are the processes $q H \rightarrow q g$ and $H \rightarrow g q \bar{q}$ with a thermal quark which contribute to the term proportional to $\left\langle T_{f q}^{00}\right\rangle$. In addition, the virtual quark-loop correction to $H \rightarrow g g$ is also proportional to $\left\langle T_{f q}^{00}\right\rangle$.

The physical picture behind obtaining (15) from the trivial insertion of (10) into (7) is that the hierarchy $M_{H} \gg T$ allows for a separation of short and long distance physics. The Higgs gluon coupling $C_{H g}$ describes physics which takes place on length and timescales much shorter than $1 / T$ and which is therefore not affected by the presence of the QCD plasma. The long-distance physics is given by the OPE of the bulk channel spectral function whose temperature dependence is parametrized by the thermal expectation values of the quark and gluon condensates, $\left\langle T_{f q}^{00}\right\rangle$ and $\left\langle T_{g}^{00}\right\rangle$, respectively. To leading order in $\alpha_{\mathrm{s}}$, these are given by the free (Stefan-Boltzmann) limits

$$
\begin{gathered}
\left\langle T_{g}^{00}\right\rangle=\frac{\pi^{2} T^{4}}{15} d_{A}, \\
\left.\left\langle T_{f q}^{00}\right\rangle\right|_{m_{q}=0}=\frac{7 \pi^{2} T^{4}}{60} d_{F},
\end{gathered}
$$

where $d_{A}=N_{c}^{2}-1$ and $d_{F}=N_{c}$ are the dimensions of the adjoint and the fundamental representation, respectively. If a quark has mass $m_{q} \lesssim T$ one would need the explicit evaluation of the massive Stefan-Boltzmann integral instead of (17), while for $m_{b} \gg T,\left\langle T_{f b}^{00}\right\rangle$ is exponentially suppressed. The sum $\sum_{q \in u d s c}$ in (15) thus goes over the flavors that can be thermally excited. To arrive at a more compact expression, one may approximate this sum by an effective number $n_{f}^{T}$ of approximately massless flavors, using $3<$ $n_{f}^{T}<4$ for temperatures well above the strange quark mass and well below the bottom charm mass. For the number of flavors entering the leading coefficient $b_{0}$ of the $\beta$-function, we use $n_{f} T_{F}=\frac{5}{2}$ in (15). With this input, we obtain

$$
\delta \Gamma_{H \rightarrow g g}=-\Gamma_{H \rightarrow g g}^{\mathrm{vac}} \alpha_{\mathrm{s}} \frac{T^{4}}{M_{H}^{4}} \frac{112 \pi^{3}}{45}\left(8-n_{f}^{T}\right),
$$

for $H$-decay in the plasma rest frame.

\section{B. $\boldsymbol{H} \rightarrow \overline{\boldsymbol{q}} \boldsymbol{q}$}

The decay of the Higgs boson into a $q \bar{q}$ pair proceeds via coupling to the scalar operator $S$. For $m_{q} \ll M_{H}$, the leading order vacuum contribution to the corresponding spectral function $\rho_{S}$ is

$$
\rho_{S}^{\mathrm{vac}}\left(K^{2}\right)=\frac{d_{F} n_{f} m_{q}^{2} K^{2}}{4 \pi},
$$

and its leading (dimension-four) thermal correction reads [4]

$$
\begin{aligned}
\delta \rho_{S}(K)= & \frac{8 \alpha_{\mathrm{s}} m_{q}^{2}}{3 K^{2}} \frac{K_{\mu} K_{\nu}}{K^{2}}\left[\frac{13}{2} C_{F} T_{f}^{\mu \nu}-n_{f} T_{F} T_{g}^{\mu \nu}\right] \\
& -\frac{9 \alpha_{\mathrm{s}} m_{q}^{2} C_{F}}{K^{2}} S .
\end{aligned}
$$

Inserting the vacuum contribution (19) into (6), we reproduce for each mass state $\left(n_{f}=1\right)$ the LO vacuum branching ratio

$$
\Gamma_{H \rightarrow q \bar{q}}^{\mathrm{vac}}=\frac{d_{F} m_{q}^{2} M_{H}}{8 \pi v^{2}}
$$

which agrees with the literature [27]. [Full accounting of the massive kinematics amounts to a multiplicative factor $\left(1-4 m_{q}^{2} / M_{H}^{2}\right)^{3 / 2}$.]

Having checked in this way the consistency of the normalization of $\rho_{S}$ and (6), one can proceed to determining in the same way the thermal correction to $\Gamma_{H \rightarrow q \bar{q}}^{\mathrm{vac}}$ from $\delta \rho_{S}(K)$ in Eq. (20). In general, the evaluation of the operator (20) in the QCD plasma requires the LO thermal (StefanBoltzmann) expectation value of the chiral condensate

$$
\langle S\rangle=4 d_{F} m_{q}^{2} \int \frac{d^{3} p}{(2 \pi)^{3}} \frac{n_{F}\left(E_{p}\right)}{E_{p}},
$$

which becomes $\frac{1}{6} d_{F} m_{q}^{2} T^{2}$ for $m_{q} \ll T$. However, for the thermal corrections to $H \rightarrow b \bar{b}$ at temperature $T \ll m_{b}$, the contributions $\langle S\rangle$ and $\left\langle T_{f}^{00}\right\rangle$ in (20) are exponentially suppressed by the quark mass, and

$$
\delta \Gamma_{H \rightarrow b \bar{b}}=-\frac{4 \alpha_{\mathrm{s}} T_{F} m_{b}^{2}}{v^{2} k_{0}} \frac{3 k_{0}^{2}+k^{2}}{9 M_{H}^{4}}\left\langle T_{g}^{00}\right\rangle+\ldots
$$

where the dots stand for $\mathcal{O}\left(\exp \left[-m_{b} / T\right]\right)$ terms. Inserting the LO expression (16) for the gluon condensate, we find (for $T \ll m_{b}$ )

$$
\delta \Gamma_{H \rightarrow b \bar{b}}=-\Gamma_{H \rightarrow b \bar{b}}^{\mathrm{vac}} \alpha_{\mathrm{s}} \frac{T^{4}}{M_{H}^{4}} \frac{128 \pi^{3}}{135},
$$

for $H$-decay in the plasma rest frame.

For temperatures $T \gtrsim \mathcal{O}\left(m_{b}\right)$ or for the calculation of the partial thermal width into lighter quarks, the contributions $\langle S\rangle$ and $\left\langle T_{f}^{00}\right\rangle$ in (20) need to be included. In general, the 
thermal corrections stemming from the coupling to lighter quarks are reduced by a factor $m_{q}^{2} / m_{b}^{2}$ compared to (24). For all partial decay widths into $q \bar{q}$-pairs, the thermal correction is an $\mathcal{O}\left(\alpha_{\mathrm{s}} \frac{T^{4}}{M_{H}^{4}}\right)$ correction to the vacuum width.

\section{Thermal corrections to spectral functions: Range of validity}

Here, we shortly recall the derivation of thermal corrections to $\rho_{J}(K)$ in the OPE approach [4], and we comment on its range of validity. The starting point is the Euclidean current-current correlator $G_{E}(q)=\int d^{4} x e^{-i q \cdot x}\langle J(x) J(0)\rangle$, where we set $q=\left(0,0,0, q_{E}\right)$ for simplicity. The dispersion relation $G_{E}\left(q_{E}\right)=P\left(q_{E}\right)+\int_{-\infty}^{+\infty} \frac{d \omega}{2 \pi\left(\omega-i q_{E}\right)} \rho_{J}(\omega)$ relates $G_{E}$ to the spectral function $\rho_{J}$, with $P\left(q_{E}\right)$ a polynomial in $q_{E}$. As explained in detail in Ref. [4], this dispersion relation implies that the asymptotic expansion of $\rho_{J}(K)$ for large timelike $K$ can be obtained from matching term-by-term to the operator product expansion of $G_{E}\left(q_{E}\right)$ for large spacelike $q_{E}$,

$$
\begin{aligned}
G_{E}\left(q_{E}\right) & \sim \sum_{n}\left\langle\mathcal{O}_{n}\right\rangle \frac{c_{n}}{q_{E}^{d_{n}}} \\
& \Longleftrightarrow \\
\rho_{J}\left(k_{0}\right) & \sim \sum_{n}\left\langle\mathcal{O}_{n}\right\rangle 2 \operatorname{Im}\left[\frac{c_{n}}{\left(-i k_{0}\right)^{d_{n}}}\right] .
\end{aligned}
$$

In practice, one proceeds as follows: First, expand the operator product $J J$ in $G_{E}(q)$ up to order $1 / q^{2}$ in the Euclidean four-momentum squared. This results in explicit expressions such as $\left.G_{E}^{\zeta}(q) \sim 4 b_{0}^{2} \alpha_{\mathrm{s}}^{2} \frac{q_{\mu} q_{\nu}}{q^{2}}\left\langle T_{g}^{\mu \nu}\right\rangle+\frac{1}{g^{2}}\left\langle\mathcal{O}_{H g}\right\rangle\right)$ for the bulk viscous channel. Then take into account that the local operators in this expansion are scale dependent, for instance, $T_{g}^{\mu \nu}\left(q_{E}\right) \sim T_{g}^{\mu \nu}\left(\mu_{0}\right)+\frac{\alpha_{s}}{3 \pi} \log \left[\frac{\mu_{0}^{2}}{q_{E}^{2}}\right]\left(n_{f} T_{F} T_{g}^{\mu \nu}\left(\mu_{0}\right)-\right.$ $\left.2 n_{f} T_{f}^{\mu \nu}\left(\mu_{0}\right)\right)$. This renormalization group flow is of central importance, since the branch cuts of the analytically continued logarithms $\log \left[\frac{\mu_{0}^{2}}{(-i K)^{2}}\right]$ contribute to $\operatorname{Im} G_{E}(-i K)$ and thus to $\rho_{J}$ at large timelike momenta. Without this RG flow, the expansion of $G_{E}(q)$ would contain only powers of the type $1 / q^{n}$ times local operators. The analytic continuation of these $1 / q^{n}$-terms to Minkowksi space can only generate discontinuities on the light cone. The only contribution to $\rho_{J}(K)$ at large timelike $K$ thus comes from these analytically continued logarithms.

The OPE of $G_{E}$ in (25) implements a physical scale separation. For a highly energetic, short-distance probe that tests distances of size $1 / q_{E}$ much smaller than any other scale in the problem, $1 / q_{E} \ll 1 / T$, Eq. (25) systematically expands in powers of that small scale times local operators. For the corresponding spectral function $\rho_{J}(K)$ to be valid, it is thus a necessary condition that

$$
K^{2}=4 k^{+} k^{-}=M^{2} \gg T^{2},
$$

where we have introduced the light-cone momenta $k^{+}=\frac{1}{2}\left(k_{0}+k\right), k^{-}=\frac{1}{2}\left(k_{0}-k\right)$. In a thermal medium and for a very massive probe, $k^{+} \gg T$ is always satisfied. However, to a boosted probe, the medium appears Lorentzcontracted, and the scale separation between the longdistance physics of the medium and the short-distance physics of the probe becomes questionable when the coherence length $\sim 1 / k^{-}$of the probe becomes comparable to the medium scale $1 / T$. One should therefore distinguish the following kinematic regimes:

(1) $k^{-} \gg T: \rho_{J}$ can be determined from OPE.

(2) $k^{-} \sim T: \rho_{J}(K)$ cannot be determined from OPE, but unresummed perturbative techniques such as those used in Refs. [25,28-30] apply for 'hard' momenta $k^{-} \sim \mathcal{O}(T)$.

(3) $k^{-} \ll T$ : Resummed finite temperature perturbation theory or nonperturbative methods would be needed to determine $\rho_{J}(K)$ in this regime, as in [31-33].

For the Higgs boson decay discussed in this section, $k^{-} \gg$ $T$ applies as long as the three-momentum $k$ in the medium satisfies $k \ll \frac{M_{H}^{2}}{4 T}$. For temperature $T \leq 1 \mathrm{GeV}$ that may be reached in heavy ion collisions at present or future colliders, the OPE and the results for the partial thermal widths (15) and (23) of the Higgs boson that we derived from it are thus valid over a transverse momentum range that extends to multiples of the Higgs mass. Over this range of validity of the OPE, thermal corrections to $\Gamma_{H}$ are seen to increase by a factor $\frac{3 k_{0}^{2}+k^{2}}{3 M_{H}^{2}}$ with the Higgs three-momentum $k$. Finally, we note that the unresummed perturbative calculations of spectral functions of the kind being considered here for $M \gtrsim T$ find that the OPE regime sets in when $M$ is approximately an order of magnitude larger than $T$ [25,28-30]. As $M_{H}$ is two orders of magnitude larger than the temperatures of QCD plasmas, the applicability of the OPE expansion is thus certain.

\section{CONCLUSIONS}

For a Higgs boson at rest in a QGP of temperature $T \ll M_{H}$, explicit expressions for the thermal corrections to the partial decay widths $\Gamma_{H \rightarrow g g}^{\mathrm{vac}}$ and $\Gamma_{H \rightarrow q \bar{q}}^{\mathrm{vac}}$ are given in Eqs. (18) and (24). These corrections are $\mathcal{O}\left(\alpha_{\mathrm{s}}\left(\frac{T}{M_{H}}\right)^{4}\right)$ times the vacuum branching ratios.

For a Higgs boson propagating with finite threemomentum $k$ through the QGP, the thermal width increases with $k$ like $\delta \Gamma^{k=0} \times\left(1+\frac{4}{3} \frac{k^{2}}{M_{H}^{2}}\right)$. This applies for $k^{-}=$ $\frac{1}{2}\left(k_{0}-k\right) \gg T$, a range of validity which includes for temperatures $T<1 \mathrm{GeV}$ even moderately relativistic Higgs bosons in the QGP.

In general, the $\mathcal{O}\left(\alpha_{\mathrm{s}}\left(\frac{T}{M}\right)^{4}\right)$ leading thermal corrections to the decay width of neutral massive particles is caused by 
the absence of lower-dimension gauge-invariant local operators in QCD. For the thermal width of the Higgs, the $T^{4}$-dependence arises from the Stefan-Boltzmann limits of the quark (17) and gluon (16) condensates that enter thermal corrections of the spectral functions (10) and (20) of the bulk viscous and scalar operator, respectively. Similarly, thermal corrections to the width of the Z-boson are $\mathcal{O}\left(\alpha_{\mathrm{s}}\left(\frac{T}{M_{\mathrm{Z}}}\right)^{4}\right)$, since the spectral functions of the vector and axial vector currents receive the dominant thermal corrections from the same quark and gluon condensates, see Ref. [4] and Appendix B.

We note that the leading $T$-dependence can be larger in theories with lower-dimension gauge-invariant local operators. In the heavy sterile neutrino case mentioned before, the zero-temperature decay into a Higgs scalar and a SM lepton receives an $\mathcal{O}\left(\lambda\left(\frac{T}{M}\right)^{2}\right)$ correction [17-19]. This is due to the dimension-two $\phi^{\dagger} \phi$ condensate of the Higgs field, with its self-coupling $\lambda$.

\section{ACKNOWLEDGMENTS}

We thank D. d'Enterria, C. Loizides, P. Nason, G. Salam and G. Zanderighi for discussions at an early stage of this work.

\section{APPENDIX A: NON-OPE RESULTS FOR THE $H \rightarrow g g$ COUPLING AT NLO}

As mentioned in the Introduction, explicit perturbative calculations of thermal widths proceed by calculating IRregulated thermal corrections to real emission, real absorption and virtual terms in the branching process. It is only the sum of these three contributions that is physically meaningful and IR-safe. The present Appendix provides technical details of how $\delta \rho_{\mathcal{O}_{H g}}(K)$, used to calculate $\delta \Gamma_{H \rightarrow g g}$ in (15), can be understood as arising from the sum of these three IR-sensitive contribution. These details are not needed to follow our derivation of thermal widths. We include them solely since they may help to understand the relation between the OPE approach followed here, and explicit perturbative calculations of thermal widths. The following discussion is limited to the pure glue part of $\delta \rho_{\mathcal{O}_{H g}}(K)$, and to $k=0$. It starts from the detailed calculation of the NLO bulk viscous spectral function, given in Ref. [25] for pure Yang-Mills theory for $k_{0} \gtrsim T$. Its applicability is hence wider than the $k_{0} \gg T$ region, and it provides a derivation of thermal corrections to the spectral function that is logically independent of the OPE and that verifies the results of the OPE.

Reference [25] calculates $\rho_{\mathcal{O}_{H q}}(K)$ at NLO in the imaginary-time formalism of thermal perturbation theory. To this end, the contributions to the $T^{\mu}{ }_{\mu} T^{\nu}{ }_{\nu}$ correlator are written without performing the sum integrals, and the sum of the amplitudes is reduced to a set of master two-loop amplitudes. One then performs first the Matsubara sums, then one analytically continues the external Euclidean frequency $k_{n}$ to the Minkowskian $k_{0}+i \epsilon$, and one finally takes the imaginary part to obtain the spectral function.

Taking this imaginary part corresponds to taking the sum over all possible cuts. At this stage, identifying the different real and virtual cut contributions to the spectral function requires introducing an IR-regulator for the soft and collinear divergences in the cuts. Different regularization schemes are possible. In Ref. [25], the authors supplement one of the propagators in the master amplitudes with a regulating mass term $\lambda$. Once the regulator has been introduced, each cut of each master amplitude is reduced to a set of one- or two-dimensional integrals. Upon summing the cuts, the $\lambda$-dependence disappears and the integrals are evaluated numerically. The final physical result is scheme independent, and thus finite, for $\lambda \rightarrow 0$.

Here, we reverse-engineer the last step of this calculation. In the Appendices A and B of Ref. [25], the real cuts are called "phase-space integrals" and the virtual ones "factorized integrals." For each cut, they can be evaluated after subtraction of the vacuum contribution for $k_{0} \gg$ $T \gg \lambda$. In this limit, many terms become exponentially suppressed $\left(\exp \left(-k_{0} / T\right) \approx 0\right)$. In particular, all two-dimensional integrals are exponentially suppressed, and one has to deal only with the easier one-dimensional ones, which we can integrate analytically for $k_{0} \gg T \gg \lambda$.

For the virtual contribution, one obtains in this way in the scheme of [25] and taking the normalization of $\mathcal{O}_{\mathrm{Hg}}$ into account

$$
\begin{aligned}
\left.\delta \rho_{\mathcal{O}_{H g}}\left(k_{0}, \lambda\right)\right|_{\text {virt }}= & \frac{d_{A} g^{2} N_{c}}{32 \pi}\left\{-\frac{k_{0}^{4}}{2 \pi^{2}}\left[\frac{2 \pi T}{\lambda}-\ln ^{2}\left(\frac{\pi T}{\lambda}\right)+2\left(\ln (4)-\gamma_{E}\right) \ln \left(\frac{\lambda}{4 \pi T}\right)+2 \gamma_{1}-\frac{\pi^{2}}{6}-\ln ^{2}(4)\right]\right. \\
& -\frac{k_{0}^{3} T}{8}-\frac{k_{0}^{2} T^{2}}{6}\left[-144 \ln (A)+4 \ln \left(\frac{64 \pi^{3} k_{0} T^{3}}{\lambda^{4}}\right)+11\right] \\
& \left.+\frac{16 T^{4}}{45 \pi^{2}}\left[\pi^{4}\left(-3 \ln \left(\frac{k_{0} T}{\lambda^{2}}\right)+3 \gamma_{E}-5-\ln (8)\right)-270 \zeta^{\prime}(4)\right]+\mathcal{O}\left(\frac{T^{5}}{k_{0}}\right)\right\},
\end{aligned}
$$

where $\gamma_{E}$ is the Euler-Mascheroni constant, $\gamma_{1}$ is the first Stieltjes constant and $\ln (A)=1 / 12-\zeta^{\prime}(-1)$ is the logarithm of Glaisher's constant. 
The real emission contribution $(H \rightarrow g g g)$ is instead

$$
\begin{aligned}
\left.\delta \rho_{\mathcal{O}_{H g}}\left(k_{0}, \lambda\right)\right|_{\mathrm{emi}}= & \frac{d_{A} g^{2} N_{c}}{32 \pi}\left\{\frac{k_{0}^{4}}{4 \pi^{2}}\left[\frac{2 \pi T}{\lambda}-\ln ^{2}\left(\frac{\pi T}{\lambda}\right)+2\left(\ln (4)-\gamma_{E}\right) \ln \left(\frac{\lambda}{4 \pi T}\right)+2 \gamma_{1}-\frac{\pi^{2}}{6}-\ln ^{2}(4)\right]\right. \\
& +\frac{k_{0}^{3} T}{8 \pi^{2}}\left[\ln ^{2}\left(\frac{2 k_{0} T}{\lambda^{2}}\right)-10 \ln \left(\frac{T}{\lambda}\right) \ln \left(\frac{4 T}{\lambda}\right)+18 \gamma_{1}-\frac{\pi^{2}}{3}+9 \gamma_{E}^{2}-10 \ln ^{2}(2)\right] \\
& +\frac{k_{0}^{2} T^{2}}{12}\left[-144 \ln (A)+4 \ln \left(\frac{64 \pi^{3} k_{0} T^{3}}{\lambda^{4}}\right)+11\right] \\
& +\frac{k_{0} T^{3}}{\pi^{2}}\left[\zeta(3)\left(\ln \left(\frac{k_{0}^{4}}{16 T^{4}}\right)+4 \gamma_{E}-15\right)-4 \zeta^{\prime}(3)\right] \\
& \left.+\frac{8 T^{4}}{45 \pi^{2}}\left[\pi^{4}\left(3 \ln \left(\frac{k_{0} T}{\lambda^{2}}\right)-3 \gamma_{E}-\frac{1}{2}+\ln (8)\right)+270 \zeta^{\prime}(4)\right]+\mathcal{O}\left(\frac{T^{5}}{k_{0}}\right)\right\} .
\end{aligned}
$$

Finally, the absorption contribution $(g H \rightarrow g g)$ reads

$$
\begin{aligned}
\left.\delta \rho_{\mathcal{O}_{H g}}\left(k_{0}, \lambda\right)\right|_{\text {abs }}= & \frac{d_{A} g^{2} N_{c}}{32 \pi}\left\{\frac{k_{0}^{4}}{4 \pi^{2}}\left[\frac{2 \pi T}{\lambda}-\ln ^{2}\left(\frac{\pi T}{\lambda}\right)+2\left(\ln (4)-\gamma_{E}\right) \ln \left(\frac{\lambda}{4 \pi T}\right)+2 \gamma_{1}-\frac{\pi^{2}}{6}-\ln ^{2}(4)\right]\right. \\
& -\frac{k_{0}^{3} T}{8 \pi^{2}}\left[\ln ^{2}\left(\frac{2 k_{0} T}{\lambda^{2}}\right)-10 \ln \left(\frac{T}{\lambda}\right) \ln \left(\frac{4 T}{\lambda}\right)+18 \gamma_{1}-\frac{4 \pi^{2}}{3}+9 \gamma_{E}^{2}-10 \ln ^{2}(2)\right] \\
& +\frac{k_{0}^{2} T^{2}}{12}\left[-144 \ln (A)+4 \ln \left(\frac{64 \pi^{3} k_{0} T^{3}}{\lambda^{4}}\right)+11\right] \\
& -\frac{k_{0} T^{3}}{\pi^{2}}\left[\zeta(3)\left(\ln \left(\frac{k_{0}^{4}}{16 T^{4}}\right)+4 \gamma_{E}-15\right)-4 \zeta^{\prime}(3)\right] \\
& \left.+\frac{8 T^{4}}{45 \pi^{2}}\left[\pi^{4}\left(3 \ln \left(\frac{k_{0} T}{\lambda^{2}}\right)-3 \gamma_{E}-\frac{1}{2}+\ln (8)\right)+270 \zeta^{\prime}(4)\right]+\mathcal{O}\left(\frac{T^{5}}{k_{0}}\right)\right\} .
\end{aligned}
$$

Upon summing the three contributions all divergent terms, as well as all terms larger than $\mathcal{O}\left(T^{4}\right)$, cancel out, yielding

$$
\begin{aligned}
\left.\delta \rho_{\mathcal{O}_{H g}}\left(k_{0}\right)\right|_{\mathrm{tot}}= & \left.\delta \rho_{\mathcal{O}_{H g}}\left(k_{0}, \lambda\right)\right|_{\mathrm{virt}}+\left.\delta \rho_{\mathcal{O}_{H g}}\left(k_{0}, \lambda\right)\right|_{\mathrm{emi}} \\
& +\left.\delta \rho_{\mathcal{O}_{H g}}\left(k_{0}, \lambda\right)\right|_{\mathrm{abs}} \\
= & -\frac{11 \pi^{2} d_{A} N_{c} \alpha_{\mathrm{s}} T^{4}}{45}
\end{aligned}
$$

If we take Eq. (10) and set $n_{f}=0, k=0$ we have

$$
\left.\delta \rho_{\mathcal{O}_{H g}}\left(k_{0}\right)\right|_{n_{f}=0}=-\alpha_{\mathrm{s}} b_{0}\left\langle T_{g}^{00}\right\rangle=-\frac{11 \pi^{2} d_{A} N_{c} \alpha_{\mathrm{S}} T^{4}}{45},
$$

which agrees as expected. The material in this Appendix further illustrates the complexity of perturbative calculations compared to the relative simplicity of deducing thermal corrections to the width from the OPE approach. The three contributions (A1), (A2) and (A3) depend, of course, on the IR regularization scheme. They illustrate, however, how the different scheme-dependent IR-singular cut contributions in a perturbative calculation sum up to a physical result that is free of any IR regulator.

\section{APPENDIX B: Z-BOSON THERMAL WIDTHS}

In between the lines of Ref. [4], one reads that it was one motivation for Caron-Huot's study of the asymptotic behavior of spectral function to clarify in a logically independent way the $\left(\frac{T}{M_{Z}}\right)$-dependence of the thermal width $\delta \Gamma_{Z}$ of the $Z$-boson for which different explicit perturbative calculations had obtained different power laws. However, despite this motivation, and despite stating clearly that the leading thermal correction in this case is $\mathcal{O}\left(\alpha_{\mathrm{s}}\left(\frac{T}{M_{Z}}\right)^{4}\right)$, the results of Ref. [4] have never been used to write an explicit expression for $\delta \Gamma_{Z}$. This Appendix aims at filling this small gap in the existing literature.

The $Z$-boson decay to $q \bar{q}$-pairs is mediated by coupling to the vector and axial vector currents

$$
\begin{aligned}
\Gamma_{Z \rightarrow q \bar{q}}= & \frac{g_{1}^{2}+g_{2}^{2}}{6 k^{0}}\left(-g_{\mu \nu}+\frac{k_{\mu} k_{\nu}}{M_{Z}^{2}}\right) \\
& \times\left[g_{V}^{2} \rho_{V}^{\mu \nu}(K)+g_{A}^{2} \rho_{A}^{\mu \nu}(K)\right]+\mathcal{O}\left(\alpha_{\mathrm{s}}\right) .
\end{aligned}
$$


Here, the vector and axial vector spectral functions $\rho_{V}$ and $\rho_{A}$ couple with $g_{V}=1 / 2 T_{3}-Q \sin ^{2} \theta_{W}, g_{A}=T_{3} / 2$, respectively, where $T_{3}= \pm 1 / 2$ for up/down-type quarks and $Q=+2 / 3$ for up-type, $Q=-1 / 3$ for down type. The factor of $1 /\left(6 k^{0}\right)$ is a combination of the usual flux factor $1 /\left(2 k^{0}\right)$ times the average over the 3 polarization states of the $Z$ boson.

For the conserved vector current we can assume $k$ to point in the $z$ direction and define $\rho_{T} \equiv \rho^{x x}=\rho^{y y}$, $\rho_{L} \equiv \frac{K^{2}}{k_{0}^{2}} \rho^{z z}=\frac{K^{2}}{k^{2}} \rho^{00}$. Hence

$$
\begin{aligned}
\Gamma_{Z \rightarrow q \bar{q}}= & \frac{g_{1}^{2}+g_{2}^{2}}{6 k^{0}}\left[g_{V}^{2}\left(2 \rho_{T}(K)+\rho_{L}(K)\right)\right. \\
& +g_{A}^{2}\left(2 \rho_{A}^{x x}(K)+\frac{k_{0}^{2}}{M_{z}^{2}} \rho_{A}^{z z}(K)+\frac{k^{2}}{M_{z}^{2}} \rho_{A}^{00}(K)\right. \\
& \left.\left.-2 \frac{k^{0} k}{M_{z}^{2}} \rho_{A}^{0 z}(K)\right)\right]+\mathcal{O}\left(\alpha_{\mathrm{s}}\right) .
\end{aligned}
$$

At vanishing quark mass the axial vector current becomes also (classically) conserved, so that we can use

$$
\rho_{T}^{\mathrm{vac}}(K)=\rho_{L}^{\mathrm{vac}}(K)=\frac{n_{f} d_{F} K^{2}}{6 \pi}+\mathcal{O}\left(\alpha_{\mathrm{s}}\right)
$$

for vector and axial current alike. This yields

$$
\Gamma_{Z \rightarrow q \bar{q}}^{\mathrm{vac}}=\frac{\left(g_{1}^{2}+g_{2}^{2}\right) n_{f} d_{F} M_{Z}^{2}}{12 \pi k^{0}}\left(g_{V}^{2}+g_{A}^{2}\right)+\mathcal{O}\left(\alpha_{\mathrm{s}}\right),
$$

which is a limit of the well-known expression for a nonnegligible mass

$$
\begin{aligned}
\Gamma_{Z \rightarrow q \bar{q}}^{\mathrm{vac}}= & \frac{\left(g_{1}^{2}+g_{2}^{2}\right) d_{F}}{12 \pi k^{0}}\left[\left(g_{V}^{2}+g_{A}^{2}\right) M_{Z}^{2}+2\left(g_{V}^{2}-2 g_{A}^{2}\right) m_{q}^{2}\right] \\
& \times \sqrt{1-\frac{4 m_{q}^{2}}{M_{Z}^{2}}}+\mathcal{O}\left(\alpha_{\mathrm{s}}\right) .
\end{aligned}
$$

For the thermal width, we need the thermal corrections to the longitudinal and transverse pieces of the vector current [4]

$$
\begin{aligned}
& \delta \rho_{T}(K)=\frac{16 \alpha_{\mathrm{s}}}{9 K^{2}} \frac{k_{0}^{2}+k^{2}}{K^{2}}\left[2 C_{F} T_{f}^{00}-n_{f} T_{F} T_{g}^{00}\right], \\
& \delta \rho_{L}(K)=\frac{16 \alpha_{\mathrm{s}}}{9 K^{2}}\left[2 C_{F} T_{f}^{00}-n_{f} T_{F} T_{g}^{00}\right] .
\end{aligned}
$$

To also obtain the corresponding thermal correction to the spectral function of the axial vector current, one can parallel for $J_{A}$ the analysis of the Euclidean $J_{V} J_{V}$ operator product in Eqs. (3.3) and (3.4) of Ref. [4]. One finds that up to dimension four, this OPE is expressed in terms of two local operators $T_{f}^{44}$ and $\mathcal{O}_{m}$, where the index 4 denotes the
Euclidean time. Since $T_{f}^{44}$ is the operator that survives in the chiral limit, it has the same Wilson coefficient in the OPE of $J_{A} J_{A}$ and $J_{V} J_{V}$. The operator $\mathcal{O}_{m}$ occurs with different Wilson coefficients in both current products, and the terms that violate current conservation are found to be proportional to it. But $\mathcal{O}_{m}$ does not matter because it is RGE invariant and does not generate cuts, so the thermal corrections to $\rho_{A}$ have to agree with those of $\rho_{V}$ even at nonzero $m_{q}$ (as long as $m_{q} \ll M_{Z}$ ). Hence the thermal correction to the $Z$ width into a quark of a particular flavor $\left(n_{f}=1\right)$ can be written as

$$
\begin{aligned}
\delta \Gamma_{Z \rightarrow q \bar{q}}= & \frac{g_{1}^{2}+g_{2}^{2}}{3 k^{0}} \frac{8 \alpha_{\mathrm{s}}\left(3 k_{0}^{2}+k^{2}\right)}{9 M_{Z}^{4}}\left(g_{V q}^{2}+g_{A q}^{2}\right) \\
& \times\left(2 C_{F}\left\langle T_{f q}^{00}\right\rangle-T_{F}\left\langle T_{g}^{00}\right\rangle\right)+\mathcal{O}\left(\alpha_{\mathrm{s}}^{2}\right) \\
= & \Gamma_{Z \rightarrow q \bar{q}}^{\mathrm{vac}} \alpha_{s} \frac{32 \pi}{27} \frac{3 k_{0}^{2}+k^{2}}{M_{Z}^{2}} \frac{2 C_{F}\left\langle T_{f q}^{00}\right\rangle-T_{F}\left\langle T_{g}^{00}\right\rangle}{M_{Z}^{4}} .
\end{aligned}
$$

For the light $u d s$ quarks in the QGP one can assume $m_{q}=0$ and take the massless expressions in Eqs. (16) and (17). One then finds $2 C_{F}\left\langle T_{f q}^{00}\right\rangle-T_{F}\left\langle T_{g}^{00}\right\rangle=\frac{2}{3} \pi^{2} T^{4}$, which yields e.g.,

$$
\delta \Gamma_{Z \rightarrow u \bar{u}}=\Gamma_{Z \rightarrow q \bar{q}}^{\mathrm{vac}} \alpha_{s} \frac{64 \pi^{3}}{81} \frac{3 k_{0}^{2}+k^{2}}{M_{Z}^{2}} \frac{T^{4}}{M_{Z}^{4}},
$$

and is identical for the branching into $d$ - and $s$-quarks. The thermal correction is again an effect of $\mathcal{O}\left(\alpha_{\mathrm{s}}\left(\frac{T}{M_{Z}}\right)^{4}\right)$, but in contrast to the standard model Higgs boson, it comes for light quarks with a positive sign.

We note that for the closely related case of dilepton production at $\frac{T}{M_{\bar{l}}} \ll 1$, the correct $\mathcal{O}\left(\alpha_{\mathrm{s}}\left(\frac{T}{M_{\bar{l}}}\right)^{4}\right)$ was found already in Ref. [9].

For the branching of the $Z$-boson into $b \bar{b}$ or $c \bar{c}$ quark pairs and for temperatures relevant for heavy-ion collision experiments, the fermion condensate in (B8) should be evaluated for massive quarks, i.e.,

$$
\left\langle T_{f}^{00}\right\rangle=4 d_{F} \int \frac{d^{3} p}{(2 \pi)^{3}} E_{p} n_{F}\left(E_{p}\right)-d_{F} \int \frac{d^{3} p}{(2 \pi)^{3}} \frac{m^{2}}{E_{p}} n_{F}\left(E_{p}\right) .
$$

For a sufficiently large ratio of quark mass over temperature, the contribution $\left\langle T_{f Q}^{00}\right\rangle$ in (B8) becomes exponentially suppressed and can be neglected. While the suppression factor $\propto \alpha_{\mathrm{s}}\left(\frac{T}{M_{\mathrm{Z}}}\right)^{4}$ will render all these effects unobservable in practice, it is still curious to note that the thermal correction $\delta \Gamma_{Z \rightarrow Q \bar{Q}}$ to sufficiently heavy quarks will be dominated by the gluon condensate and therefore have a negative sign, in contrast to (B9). 
[1] E. L. Berger, J. Gao, A. Jueid, and H. Zhang, Phys. Rev. Lett. 122, 041803 (2019).

[2] Z. Citron et al., arXiv:1812.06772.

[3] D. d'Enterria and C. Loizides, arXiv:1809.06832.

[4] S. Caron-Huot, Phys. Rev. D 79, 125009 (2009).

[5] H. B. Meyer, J. High Energy Phys. 04 (2010) 099.

[6] M. Laine, M. Vepsalainen, and A. Vuorinen, J. High Energy Phys. 10 (2010) 010.

[7] T. Inami, T. Kubota, and Y. Okada, Z. Phys. C 18, 69 (1983).

[8] R. Baier, B. Pire, and D. Schiff, Phys. Rev. D 38, 2814 (1988).

[9] T. Altherr and P. Aurenche, Z. Phys. C 45, 99 (1989).

[10] T. Altherr, P. Aurenche, and T. Becherrawy, Nucl. Phys. B315, 436 (1989).

[11] Y. Gabellini, T. Grandou, and D. Poizat, Ann. Phys. (N.Y.) 202, 436 (1990).

[12] J. I. Kapusta and S. M. H. Wong, Phys. Rev. D 62, 037301 (2000).

[13] A. Majumder and C. Gale, Phys. Rev. C 65, 055203 (2002).

[14] M. L. Bellac, Thermal Field Theory (Cambridge University Press, Cambridge, England, 1996).

[15] A. Czarnecki, M. Kamionkowski, S. K. Lee, and K. Melnikov, Phys. Rev. D 85, 025018 (2012).

[16] M. Beneke, F. Dighera, and A. Hryczuk, J. High Energy Phys. 09 (2016) 031.

[17] M. Laine and Y. Schroder, J. High Energy Phys. 02 (2012) 068.
[18] A. Salvio, P. Lodone, and A. Strumia, J. High Energy Phys. 08 (2011) 116.

[19] S. Biondini, N. Brambilla, M. A. Escobedo, and A. Vairo, J. High Energy Phys. 12 (2013) 028.

[20] S. Biondini et al., Int. J. Mod. Phys. A 33, 1842004 (2018).

[21] M. Kramer, E. Laenen, and M. Spira, Nucl. Phys. B511, 523 (1998).

[22] K. G. Chetyrkin, B. A. Kniehl, and M. Steinhauser, Nucl. Phys. B510, 61 (1998).

[23] M. Steinhauser, Phys. Rep. 364, 247 (2002).

[24] J. Grigo, K. Melnikov, and M. Steinhauser, Nucl. Phys. B888, 17 (2014).

[25] M. Laine, A. Vuorinen, and Y. Zhu, J. High Energy Phys. 09 (2011) 084.

[26] A. Djouadi, M. Spira, and P. M. Zerwas, Phys. Lett. B 264, 440 (1991).

[27] A. Djouadi, Phys. Rep. 457, 1 (2008).

[28] M. Laine, J. High Energy Phys. 05 (2013) 083.

[29] M. Laine, J. High Energy Phys. 08 (2013) 138.

[30] M. Laine, J. High Energy Phys. 11 (2013) 120.

[31] P. Aurenche, F. Gelis, G. D. Moore, and H. Zaraket, J. High Energy Phys. 12 (2002) 006.

[32] I. Ghisoiu and M. Laine, J. High Energy Phys. 10 (2014) 83.

[33] J. Ghiglieri and G. D. Moore, J. High Energy Phys. 12 (2014) 029. 